\title{
Vector Analysis of Problem Spaces Based on Human-Machine Interaction
}

\author{
Liu Changyong ${ }^{1}$, Cheng Rengui ${ }^{1}$ and Meng Shimin ${ }^{1,2^{*}}$ \\ ${ }^{I}$ Graphical Image Lab, Wuyi University, Wuyishan 354300, Fujian, China \\ ${ }^{2}$ DNAgent Lab@UlinkM.com INC., Wuyishan 354300,Fujian, China
}

\begin{abstract}
As a problem, space is the implicit world of cognition, it is difficult to observe and collect its data. From the new perspective of cognitive philosophy, human-machine cognitive coupling is the coupled state of the intelligent agent and the information system. The extension of a problem space inside the brain makes observation possible. The data for a problem space constitutes a set and forms a tree topology according to the level of cognitive abstraction. On the basis of mathematical paradigms, the metric specifications of problems are built on problem topology. Vector analysis is carried out on the problem space. The size, direction, hierarchy, and vector addition and subtraction of objects of a problem space are defined. On the basis of topological dynamic theory, the topological dynamic orbit of a problem space is defined. The topological reconstruction and vector analysis of problem spaces provides new methods for the refined detection of the structure of problem spaces.
\end{abstract}

Keywords: Cognitive dynamics system, E-learning, Human-machine interaction, Problem space, Topological manifold.

\section{INTRODUCTION}

In the late 1960s, modern cognitive psychology came into being. Problem solving was directed at high-level cognitive and intelligent activities. A number of cognitive models were proposed and represented by the problem space and problem search models proposed by Allen Newell and Herbert Alexander Simon, founders of American information processing psychology (Newell\&Simon, 1972) [1]. Currently, problem space studies are primarily carried out in the fields of cognitive psychology and pedagogy and there is The Interdisciplinary Journal of Problem-based Learning (IJPBL) (http://docs.lib.purdue.edu/ijpbl/). At present, the research background of problem spaces is cognitive psychology, and problem space studies, characterized by psychology, primarily rely on observations and investigations to collect data and use statistical methods to process data. Compared with disciplines such as physics and chemistry, psychology is often considered prescience, and improving the research methods and paradigms of psychology is the trend. It is also our goal to look for new methods for problem space studies.

In 1879, Wilhelm Wundt set up the first psychology lab at the University of Leipzig. In the initial phase of research, Wundt aimed at looking for the commonalities and trends of human psychology. However, he found that different people responded differently to the same stimulus. At the beginning, Wundt thought this was caused by errors in experimental procedures. Afterwards, through a long period of observations, he discovered that the personality difference of human psychology is ubiquitous. Therefore, Wundt began to focus on personality measurement and took measurement as the foundation of psychological studies [2]. For more than 100 years, the theories and schools of psychology have diversified due to the personality difference of psychological phenomena. We can ask, "Are there relatively stable psychomotor laws governing the psychological appearances of numerous and complex personality differences just like our universe in which the motion of the dense clusters of stars must obey laws including the law of universal gravitation and the biological world where the evolution of various animals and plants rely on genes to maintain the complexity of biological appearances and the relative stability of genes. Do forces and genes exist in the psychological world and the problem space? We hope to study problem spaces from the deep perspective of mathematical and scientific paradigms instead from the perspective of appearances in order to explore the connotations and laws of problem spaces.

Following the idea of empirical science, problem space studies need to observe problem spaces, collect the activity data for the objects of problem spaces, and establish problem space models based on tools and technology. Collecting the data for problem spaces is a research difficulty. This is because a problem space is an implicit psychological world and it is difficult for us to collect the data for the problem space using common tools and technology. Conventional cognitive science relies heavily on human means such as oral and written communication and visual observation to collect the data for problem spaces. For example, cognitive psychology experiments primarily infer the psychological spaces of cognition from language interaction, response time, and the electrical signals of brain neural activities. From the perspective of empirical science, human data collection is restricted in terms of objectivity, amount and accuracy. How to observe 
problem spaces using methods other than conventional psychological methods? For example, in the course of learning subjects, students can encounter a great number of individual problems and doubts and it is obviously difficult to rely on conventional psychological questionnaires and language surveys to satisfy the research needs of problem spaces.

According to the research paradigm of empirical science, innovative methods, technology, and tools are required to improve the observation of problem spaces. According to the history of the use of scientific research tools, as problem space is the implicit world of cognition, it is difficult to observe and collect its data. Therefore, innovating automated, intelligent observation technology and equipment for problem spaces are important means for us to improve problem space studies. This equipment replaces problem investigators to complete human affairs. Obviously, using computers to collect data instead of humans is considered. Can computers become investigators of problem spaces? Currently, computers as intelligent devices have been widely used in various fields. It is reasonable for us to believe that computers can replace humans to complete most of the investigations into the problem spaces. It is a trend for problem space and cognitive science studies to develop related software systems [3, 4] so as to enable computers to become observation equipment for problems spaces and collect the activities of students' cognitive psychological world. Following the idea of the research paradigm of empirical science, how to reconstruct the collected data for problem spaces? Or what is the structure of a problem space? From the mathematical perspective, a problem space is a high-dimensional manifold and the collected data can be reconstructed in a lowdimensional space structure, namely the dimensionality reduction of a high-dimensional problem space. Analyzing and reconstructing problem spaces based on manifold theory [5] is also an important means for us to study problem spaces.

All in all, our studies focus on observing problem spaces and collecting the activity data for the objects of problem spaces by constructing human-machine interactive systems, and looking for the low-dimensional structures of problem spaces based on manifold analysis theory so as to reconstruct problem spaces.

\section{OBSERVATION AND TOPOLOGICAL RECON- STRUCTION OF PROBLEM SPACES BASED ON COUPLED HUMAN-MACHINE COGNITION}

To observe problem spaces by using computers, it is obviously necessary to design effective human-machine interactive environment. Human-machine interactive situation is the convergence of the psychological world and the information world. To turn computers into equipment for observing problem spaces, it is necessary to solve the problems of how to observe data and obtain scientific data. It is feasible to collect data in a human-machine interaction. The collected data are the representation of the objects and motion of problem spaces. On the basis of situated, distributed, embodied and extended cognition, we propose that there are a lot of states in the interaction between the brain cognitive agent and the information environment, including the coupled state.
Coupled humans and machines form coupled cognition. On the basis of category and morphism theory, we construct a coupled human-machine cognitive mechanism and propose the coupling effect of cognitive and information spaces. That is, in the coupled state of human-machine cognition, the cognitive-information space has "cognitive natural isomorphism and cognitive natural transformation" principles. The above two principles maintain the structural isomorphism and relationship covariance of the information-cognitive space. We call them "the cognitive coupling observation principle" (CCOP). On the basis of CCOP, human-machine interactive environment breaks down into brain cognitive agent, coupling situation and information manifolds (BSM) and becomes the platform for observing problem spaces [6].

\subsection{Observation of High-Dimensional Problem Mani- folds Based on Cognitive Frames}

CCOP-based observation of problem spaces contain the following viewpoints: 1) From the perspective of category theory, the brain cognitive agent, the coupled humanmachine situation, and the information manifold are three objective carriers of cognitive objects or structures capable of cognitive computing. 2) There are cognitive morphisms between BSM. The cognitive objects and relationships inside the brain can be coupled to human-machine interaction and then to information manifolds. Problem spaces will be mapped to information manifolds. The reverse process can also be realized. 3) In BSM, there are cognitive natural isomorphism and cognitive natural transformation principles which ensure the objectivity and covariance of observation.

Problem spaces are high-dimensional manifolds. We also call problem spaces problem manifolds which are a kind of cognitive manifolds. The overall structure and local characteristics of a problem manifold cannot be measured directly. In order to study a problem manifold, it is required to be locally mapped to a Euclidean space, as shown in Fig. (1). This is the basic method of manifold analysis. For example, the earth is a spherical manifold. In manifold analysis, the earth can be locally assumed to be a flat and straight Euclidean space, namely describing the local space of the earth using the Cartesian coordinate system. There are two problems to be solved through the problem manifold analysis: how to obtain the parts of a problem manifold and how to obtain the local space of a problem manifold, namely how to measure the parts of a problem manifold and how to reconstruct a problem manifold.

In our opinion, in the BSM environment, coupled humanmachine interfaces can carry the local situation of problem manifolds. That is, in the state of cognitive coupling, humanmachine situation is the local projection of cognitive manifolds and can also become the local projection of problem manifolds. Or in other words, in the coupled human-machine environment, human-machine situation interface is the local expression of brain cognitive manifolds. For example, a picture of "panda" appears in the coupled human-machine situation. If the brain cognitive agent confirms that this interface characterizes "panda", then we think that the "panda" in the current human-machine situation is a part of the cognitive 


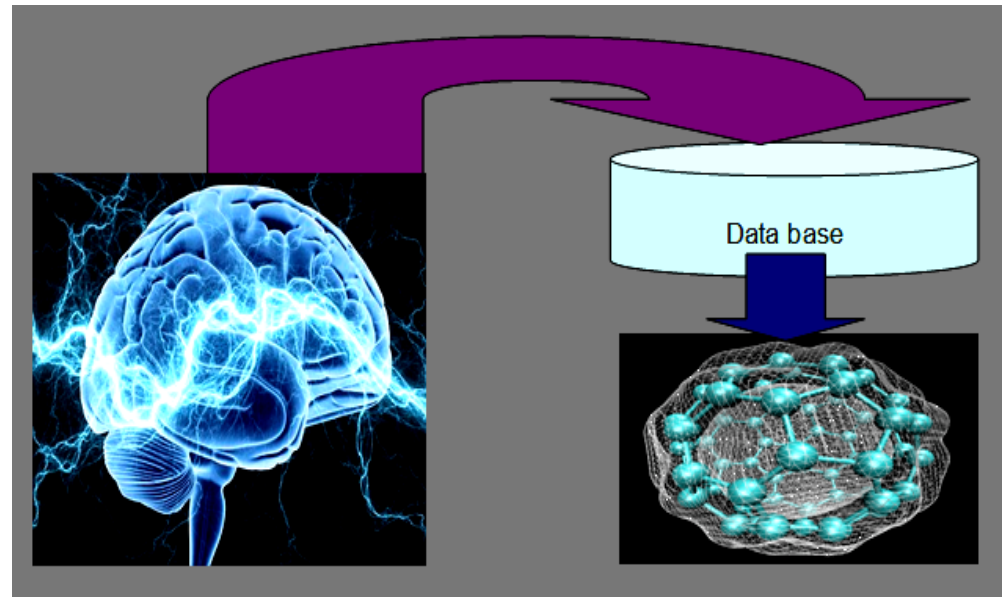

Fig. (1). Collect high-dimensional problem manifold data for reconstruction in a low-dimensional topological space.

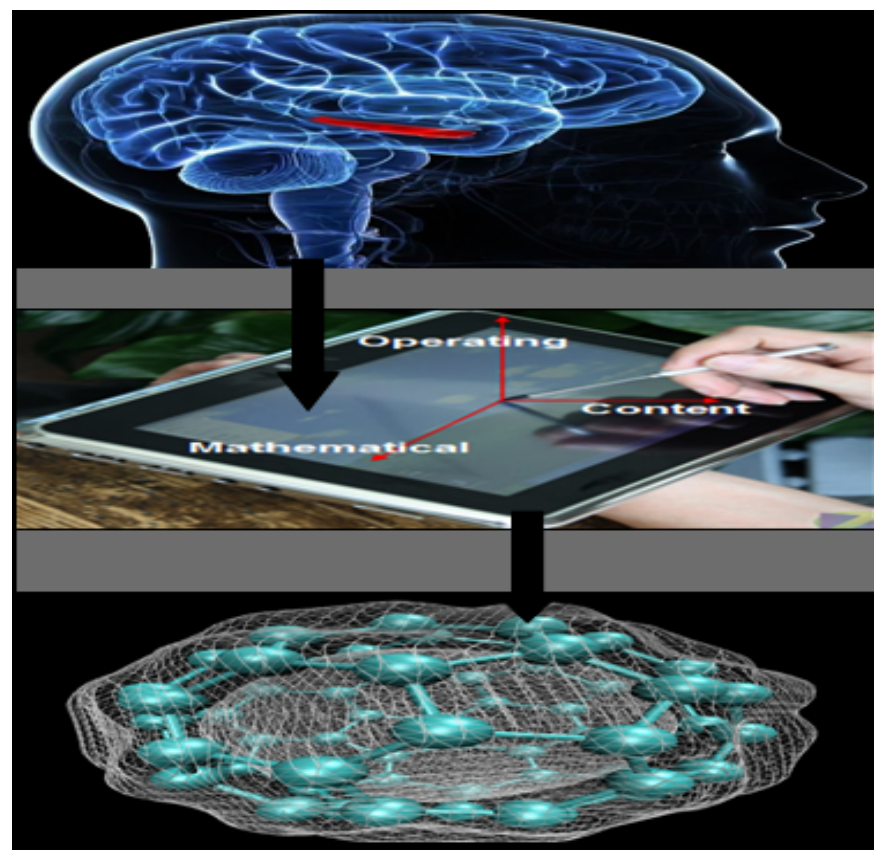

Fig. (2). Vectorization of human-machine situation based on COM frame.

manifold and problem manifold, namely a situation representation of "panda". The "panda" in the coupled humanmachine situation maps and characterizes the element and object "panda" in the cognitive manifold and problem manifold. Here, we project the objects and situations of a highdimensional problem space onto the human-machine interface through CCOP and externalize implicit cognitive content, thus providing a method for observing content-oriented brain cognition. The observation outside the brain of cognition inside the brain is also an application of cutting-edge cognitive distribution and extension.

After projecting a high-dimensional problem manifold onto human-machine situation interface, we can measure parts of this problem manifold. According to the manifold analysis theory, in order to measure parts of a problem manifold, a frame or a coordinate system is required to be established. A coordinate system is the observation perspective of human-machine interface interaction in observation of cognitive and problem manifolds as well as the dimensionality of data collection [7]. We propose the concept of cognitive frame. In order to observe cognitive situation, we need to establish a three-dimensional frame of cognitive operation, cognitive content and mathematical parameters, namely the COM frame as shown in Fig. (2). Through the COM frame, we will obtain data for human-machine interaction. These data describe cognitive human-machine interaction and are called the sequence of cognitive symbols. Based on the cognitive frame and the sequence of cognitive symbols [8], we implement the vectorization of human-machine situation and convert human-machine interaction into data and mathematical description.

All in all, after clarifying the objectivity and covariance of BSM observations, we further establish a mathematical observation system of problem spaces based on topology manifold theory and propose :1) The problem space is a high-dimensional manifold; 2) coupled human-machine interactive situation is the local measure of high-dimensional problem manifolds; 3) to vectorize human-machine 


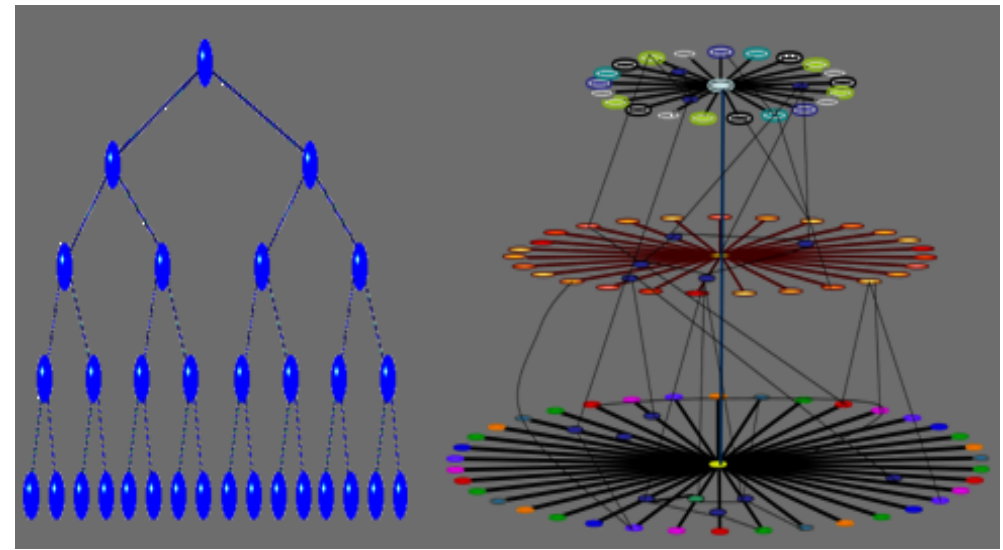

Fig. (3). Plane and three-dimensional tree topological structures of problem spaces.

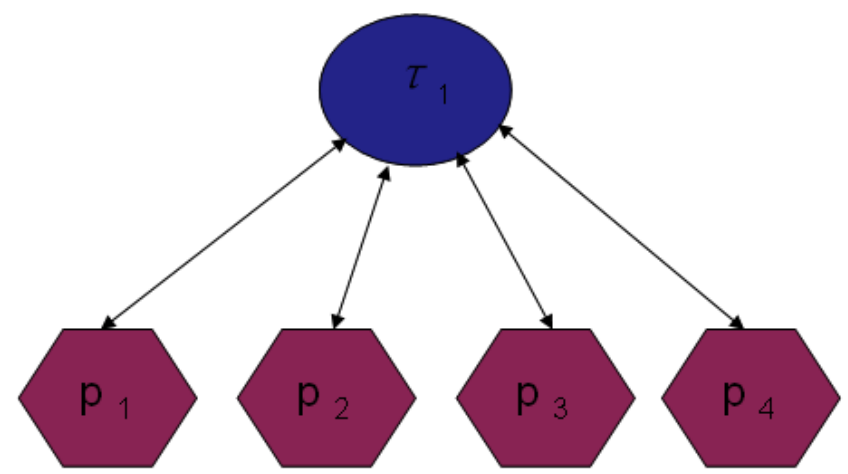

Fig. (4). Establishment of a high-order topological space based on sets.

interactive situation and collect data for cognitive symbol sequences based on the frame method; 4) to project problem manifolds onto low-dimensional cognitive metric spaces for the dimensionality reduction of high-dimensional cognition. Through coupled situation, high-dimensional problem situation is coupled to human-machine interface and then projected onto low-dimensional problem manifolds through the COM frame.

\subsection{The Low-Dimensional Structure of High- Dimensional Problem Spaces}

In the above, we analyze the observation methods for the objects of problem spaces and solve the first problem about the analysis of problem spaces. Now, we must solve the second problem, namely what is the structure of a lowdimensional problem manifold space? Simon et al. defined the structure of a task or problem space as tree structure [9], namely using hierarchical tree structure to express problem spaces, as shown in Fig. (3).

Following Simon's idea, we define tree structure as the low-dimensional structure of a problem manifold. However, we must describe tree topology using mathematical methods. Describing cognitive structure using topology theory [8] is an effective method. Assume that the problem space is a topological structure, namely $\mathrm{Q}$ is a problem set. The family of subsets $\tau$ of $Q$ is called a topology of Q. If it satisfies:
(1) Q and empty set \{\} belong to $\tau$.

(2) The union of any number of members in $\tau$ is still in $\tau$.

(3) The intersection of a limited number of members in $\tau$ is still in $\tau$.

Set $\tau$ is called a topological space if it satisfies the above three topological axioms, denoted as $(\mathrm{Q}, \tau)$. In addition to sets, hierarchies are also used to characterize problem spaces. A subset of a problem set is mapped to an element of a highorder topological set to form a hierarchy of the problem space. We call these hierarchies the "orders" of problem spaces. Hierarchies are equivalent to the height of a geometric space, as shown in Fig. (4).

From the above perspective, we analyze two problems about observation of problem manifolds or problem spaces, namely the cognitive frame observation method of problem manifolds based on coupled human-machine cognition and the low-dimensional manifold structure of problems based on tree topology, solving the observation and reconstruction problems of problem manifolds.

\section{VECTOR ANALYSIS OF TOPOLOGICAL PROB- LEM SPACES}

On the basis of BSM, we can collect accurate information about problem spaces. On the basis of the 


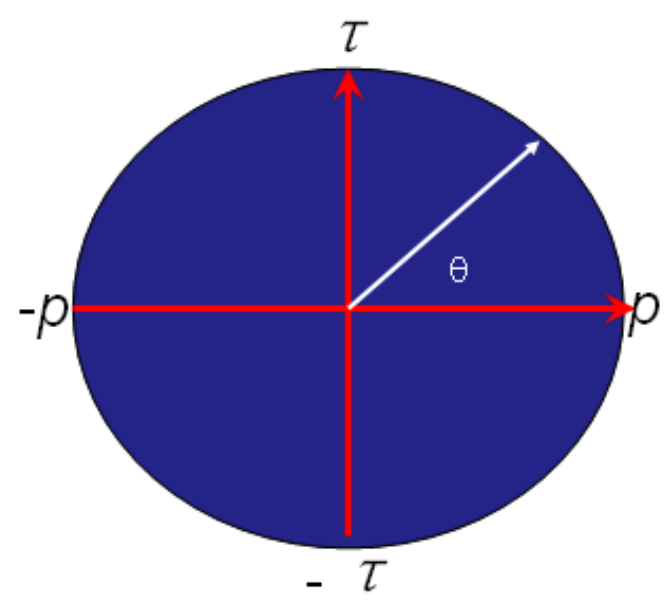

Fig. (5). The coordinate system and vector angle of the object of a problem space.

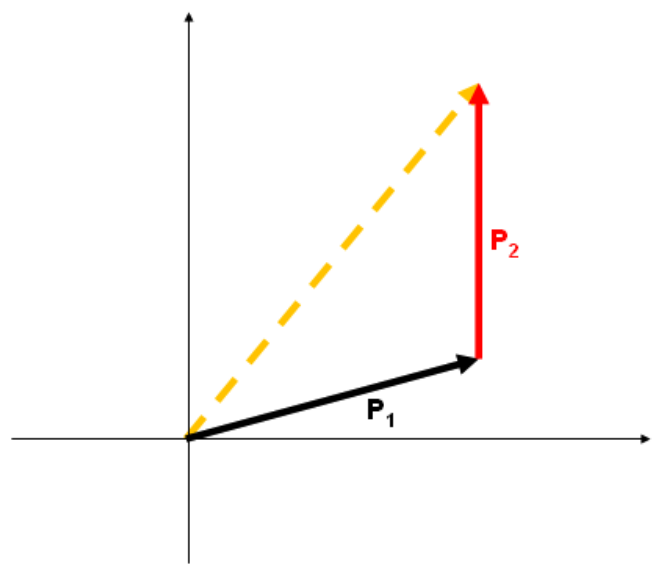

Fig. (6). The triangle law of the vector addition and subtraction of problem spaces.

research paradigm of mathematical sciences, we will add the metric characteristic to the topological problem space to form the metric problem space. In the metric space, we introduce mathematical methods such as differential dynamic systems. The basic characteristic of the metric space is cognitive vectors which represent the basic relationship and motion characteristic of the cognitive world.

In the physical world, vectors are represented by forces. Forces are interactions between objects. The basic attributes of forces are the direction, the size, and the point of action. In the cognitive world and the problem space, problem objects also have correlation which we call the cognitive force. Similarly, the cognitive force also has the direction, the size, and the action of point. In the problem space, the cognitive force is primarily reflected by the logic trend, intuitive relationship, and object sorting, etc. reflected in the course of problem understanding and searching.

Definition 1. Quantity of Cognitive Force: Assume that the object of a topological problem space is $\mathrm{P}$. There is a relationship between $P_{1}$ and $P_{2}$. The relationship from $P_{1}$ to $\mathrm{P}_{2}\left(\mathrm{P}_{1} \rightarrow \mathrm{P}_{2}\right)$ is represented by $\mathrm{C}_{12}$ and on the contrary, the relationship from $\mathrm{P}_{2}$ to $\mathrm{P}_{1}\left(\mathrm{P}_{2} \rightarrow \mathrm{P}_{1}\right)$ is represented by $\mathrm{C}_{21}$.

Definition 2. Direction of Cognitive Force: In a problem space, the number of correlations between the object $\mathrm{P}$ and other objects is the degree of correlation of $\mathrm{P}$. If $\mathrm{P}$ is not correlated with any other object, then its degree of correlation is $0 . \mathrm{P} \rightarrow \mathrm{P}_{1}, \mathrm{P} \rightarrow \mathrm{P}_{2}, \mathrm{P} \rightarrow \mathrm{P}_{3} \ldots \mathrm{P} \rightarrow \mathrm{P}_{\mathrm{n}}$ represent $\mathrm{P}$ 's degree of correlation $\mathrm{M}=\mathrm{n} ; \mathrm{P} \leftarrow \mathrm{P}_{1}, \mathrm{P} \leftarrow \mathrm{P}_{2}, \mathrm{P} \leftarrow \mathrm{P}_{3} \ldots \mathrm{P} \leftarrow \mathrm{P}_{\mathrm{n}}$ represent $\mathrm{P}$ 's degree of correlation $\mathrm{M}=-\mathrm{n}$.

Definition 3. Direction Angle of Cognitive Force $\theta$ : According to the definition of topology, we have known that the subset $\left\{\rho_{1}, \rho_{2}, \rho_{3}, \rho_{4}\right\}$ of set $P$ is mapped to an element $\tau_{1}$ in the topological space $\tau$ and defines that $\tau$ is an order higher than $\mathrm{P}$ so as to form a concept similar to the height of a Euclidean space. Therefore, it can be considered that $\mathrm{P}$ and $\tau$ are vertical, namely $90^{\circ}$. - $p$ and $-\tau$ respectively represents correlation logic from inside to outside, or topology from the upper layer or the lower layer, as shown in Fig. (5).

From the above perspective, in a problem space, a learner has no doubts about the relevant elements of the object in the same order as object $\mathrm{P}$, the object in a higher order, and the object in a lower order. On the contrary, if there are any doubts about an object $\mathrm{P}$ of the problem space and they are points that cannot be understood and are encountered during search, these points may appear in P's same-order, higherorder or lower-order elements.

Definition 4. Vector Addition of Cognitive Force: The cognitive vectors $\mathrm{P}_{1}$ and $\mathrm{P}_{2}$ of two problem spaces add to obtain their vector sum $\mathrm{P}_{12}$, as shown in Fig. (6). 


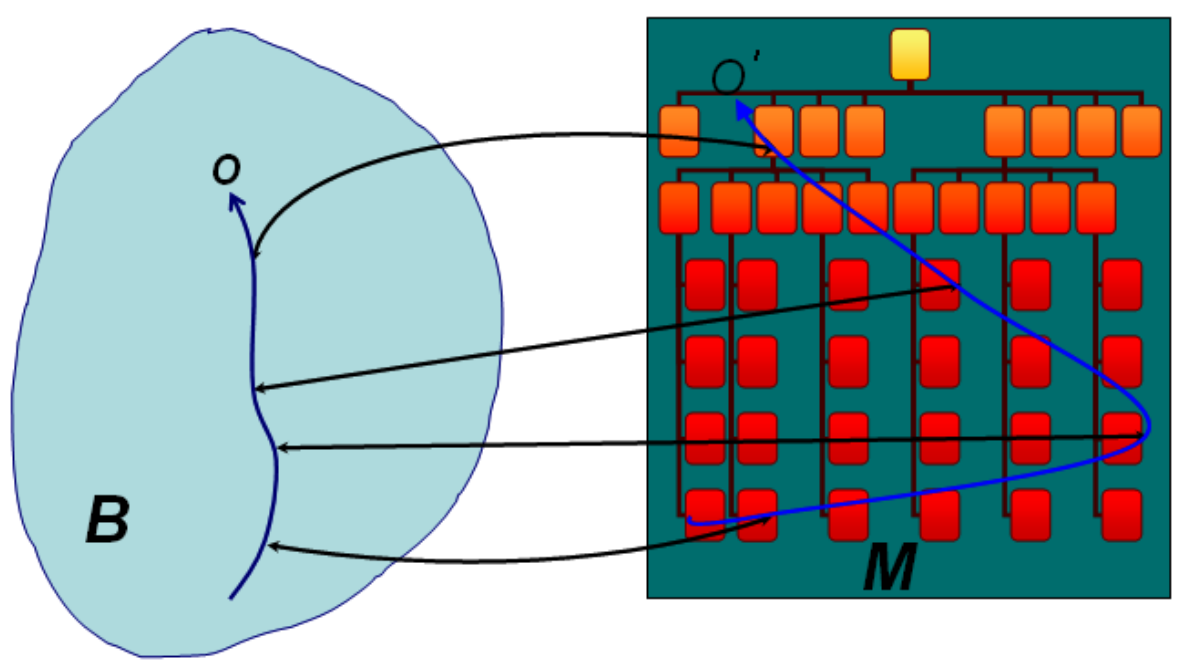

Fig. (7). The schematic diagram of the projection of a cognitive orbit.

Definition 5. Cognitive Orbit: In the problem solving process, searching problem spaces based on goals is a basic process. We project the objects involved in problem search onto low-dimensional manifolds and make these problems and cognitive objects into sequence $\mathrm{O}\left(\mathrm{O}_{1}, \mathrm{O}_{2}, \mathrm{O}_{3}, \ldots \mathrm{O}_{\mathrm{n}}\right)$ so as to observe the orbit of problem search, as shown in Fig. (7).

The key to the observation of problem search orbits lies in designing the structure of low-dimensional cognitive manifolds and selecting cognitive frames to collect problem search data. For example, we project problem search onto the tree space shown in Fig. (7) and develop related metric specifications so as to develop a problem search vector space. On the basis of mathematical specifications, we can mathematically process low-dimensional cognitive manifolds. For example, assume that all points in a tree space are uniformly measured. For example, 1) the measures of time $t$ are unified, and time is an integral parameter or a variable; 2 ) if a variable $K$ is a function of $t$, namely $K=f(t)$, and different units of measurement of $\mathrm{K}$ can be added, then 3 ) we can perform mathematical operations such as integration of $\mathrm{K}$ of orbit $\mathrm{O}$ based on $\mathrm{t}_{\mathrm{a}} \rightarrow \mathrm{t}_{\mathrm{b}}$ :

$E=\int_{t_{a}}^{t_{b}} f(t) d t$

With powerful mathematical tools such as calculus, we can carry out effective cognitive dynamic studies.

On the basis of topological problem spaces, we vectorize the relationship of problem objects to develop a mechanical system like physics. Although this is superficial, it has provided support for our mathematical and empirical problem space studies. According to the manifold theory, an $\mathrm{N}$-dimensional manifold can be embedded into a $(\mathrm{N}+1)$ dimensional Euclidean space. Similarly, we can embed a tree problem space into a three-dimensional Euclidean space and use our familiar methods for three-dimensional spaces to process problem spaces and cognitive vectors.

\section{VECTORIZATION EXAMPLE OF PROBLEM SPACES}

\subsection{Vectorization of Problem Spaces Based on Cognitive Frames}

Conventional problem space studies primarily rest on thinking. In a broad sense, when students learn a course, the knowledge and skills of the course are mapped into their cognitive psychological world to form a broad problem space. In the course of learning, students' understanding of knowledge is to constantly contact objects, activate situations and search for goals in the problem space. The search and contact in the problem space can encounter barriers, doubts and faults which interrupt search. These interruptions directly affect students' understanding of the course and are also the effective information required to be understood in teacher's teaching. We collect the barriers and doubts of problem spaces based on courses and schools.

The experimental process is as follows:

(1) Scan a textbook so as to form the collection situation of the problem space.

(2) Students raise questions about their doubts in the electronic textbook in the human-machine interactive environment. The icons in Fig. (8) represent students' doubts about the knowledge and content in the picture.

(3) Collect and record students' question information based on the cognitive frame.

(4) Preliminarily screen the contents of the questions and sum up the contents into a problem set. Table $\mathbf{1}$ is the problem set we collect.

(5)Classify the problem set into different problem subsets.

(6) Problem subsets are abstracted and mapped into a high-order problem set. A topological space is reconstructed based on the high-order problem set. Fig. (9) is the high-order 


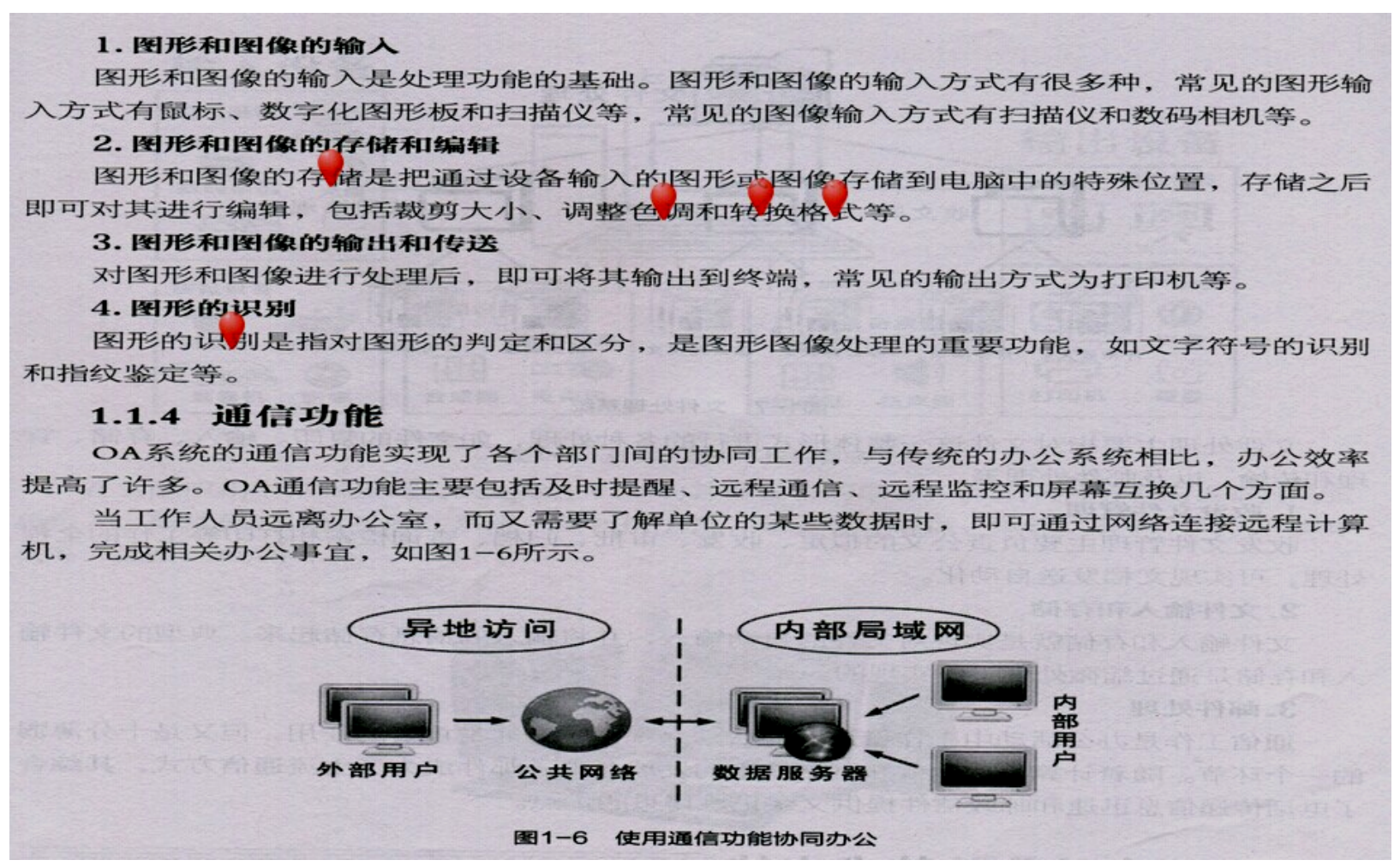

Fig. (8). Textbook situation marking.

Table 1. Students' problem set.

\begin{tabular}{|c|c|c|c|c|c|c|}
\hline Name & Gender & Subject & Content & $\begin{array}{l}\text { First-order } \\
\text { category }\end{array}$ & $\begin{array}{l}\text { Second-order } \\
\text { category }\end{array}$ & $\begin{array}{c}\text { Classification of } \\
\text { Chapters }\end{array}$ \\
\hline Su Chen & Male & $\begin{array}{l}\text { Customization of } \\
\text { data screening }\end{array}$ & $\begin{array}{c}\text { What are the results of custom "and" } \\
\text { and "or" }\end{array}$ & Screening & Concept & EXCEL \\
\hline Wang Quantao & Male & Chart data & $\begin{array}{c}\text { What are the key points in updating } \\
\text { chart data }\end{array}$ & Chart data & Concept & EXCEL \\
\hline Wang Quantao & Male & Create a chart & $\begin{array}{l}\text { What are the details about creating a } \\
\text { chart }\end{array}$ & Create charts & Concept & EXCEL \\
\hline Wang Zhijie & Male & Cell & $\begin{array}{l}\text { What is the cause of the error value \# } \\
\text { REF! in cells? How to solve this? }\end{array}$ & Errors in cells & Concept & EXCEL \\
\hline Wang Zhijie & Male & Cell & $\begin{array}{l}\text { What is the cause of the error value } \\
\text { \#VALUE in cells? How to solve this? }\end{array}$ & Errors in cells & Concept & EXCEL \\
\hline Wang Zhijie & Male & Workbook & The basic operation of workbooks? & $\begin{array}{l}\text { Basic operation } \\
\text { of workbooks }\end{array}$ & Concept & EXCEL \\
\hline Wang Zhijie & Male & Subtotal & How to operate a PivotChart? & PivotChart & Concept & EXCEL \\
\hline Xiao Shilong & Male & Subtotal & The steps and details of subtotals & Subtotal & Concept & EXCEL \\
\hline Xiao Shilong & Male & $\begin{array}{l}\text { Formula compu- } \\
\quad \text { tation }\end{array}$ & $\begin{array}{l}\text { What is a formula? How does it func- } \\
\text { tion? Which are operators? Which are } \\
\text { parameters for formula operations? }\end{array}$ & formula & Concept & EXCEL \\
\hline
\end{tabular}




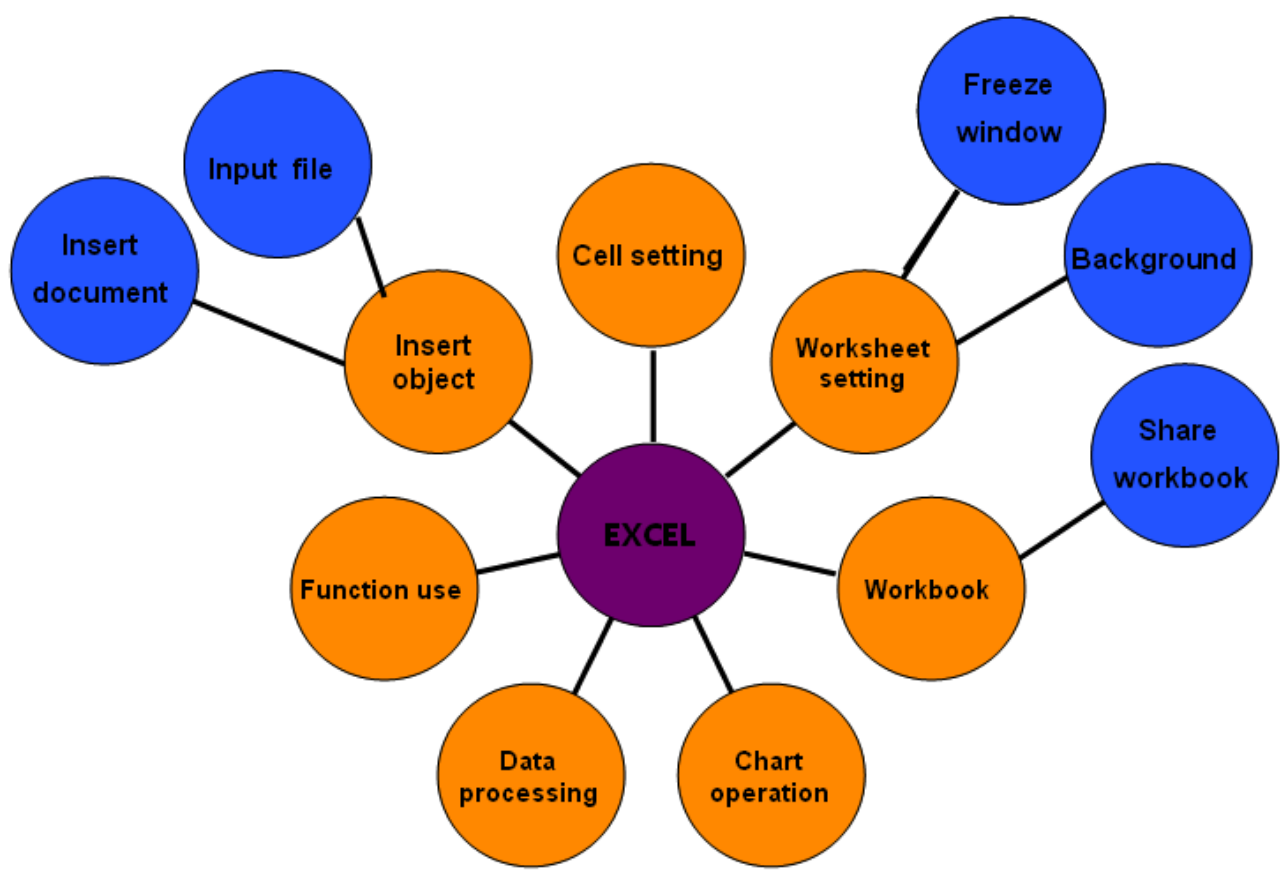

Fig. (9). Construction of a first-order problem topology $(\mathrm{Q}, \tau)$ of EXCEL based on set Q.

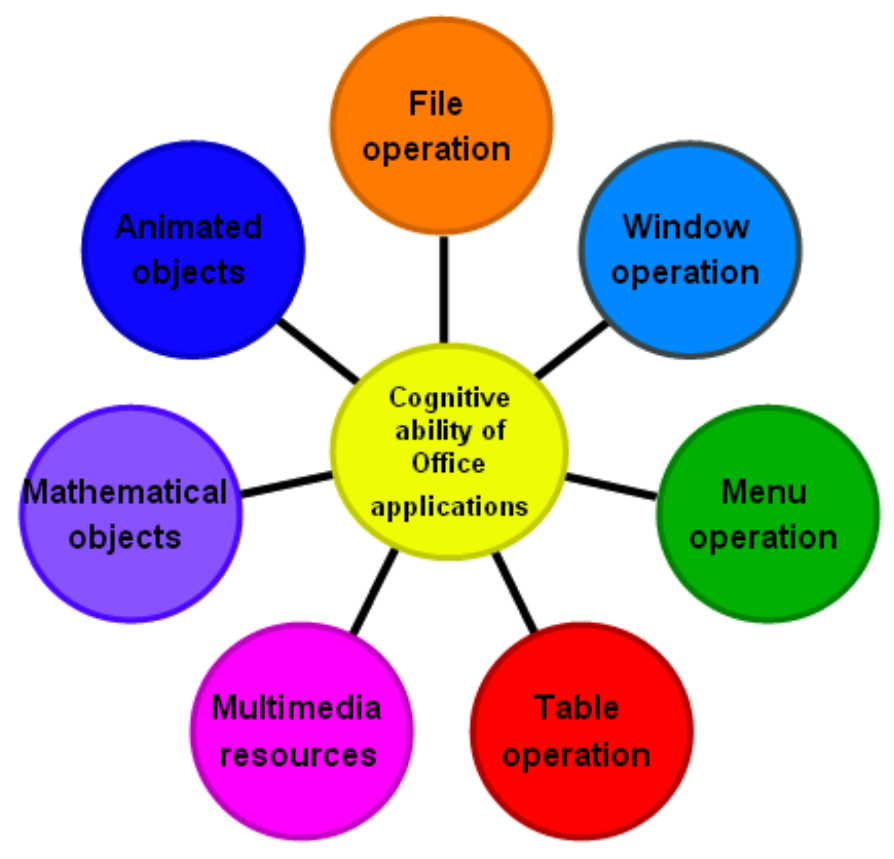

Fig. (10). Construction of a second-order problem topology $(K, \varnothing)$ of Office based on set K.

topological space we construct on the basis of the contents of the textbook.

(7) According to our needs, abstraction and topologization are performed. As shown in Fig. (10), we further construct a high-order topological space based on the topological structure shown in Fig. (9).

From the above perspective, we demonstrate how to construct and vectorize problem spaces, collect data for problem objects to establish cognitive sets, analyze the collected data based on manifold topology analysis, and construct topological problem spaces.

\subsection{Vectorization Observation of Problem Objects Based on Testing Environment}

In human-machine interactive learning testing, students solve problems based on testing situation. We will be able to vectorize this situation and obtain the learning states of cognitive objects. 


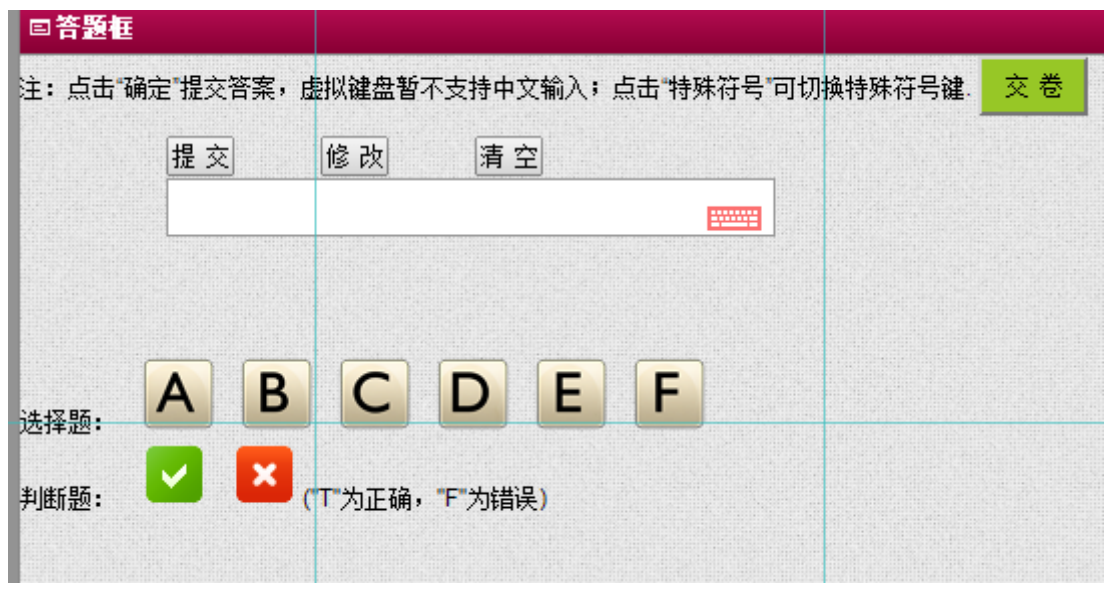

Fig. (11). Human-machine interface for collecting test operation.

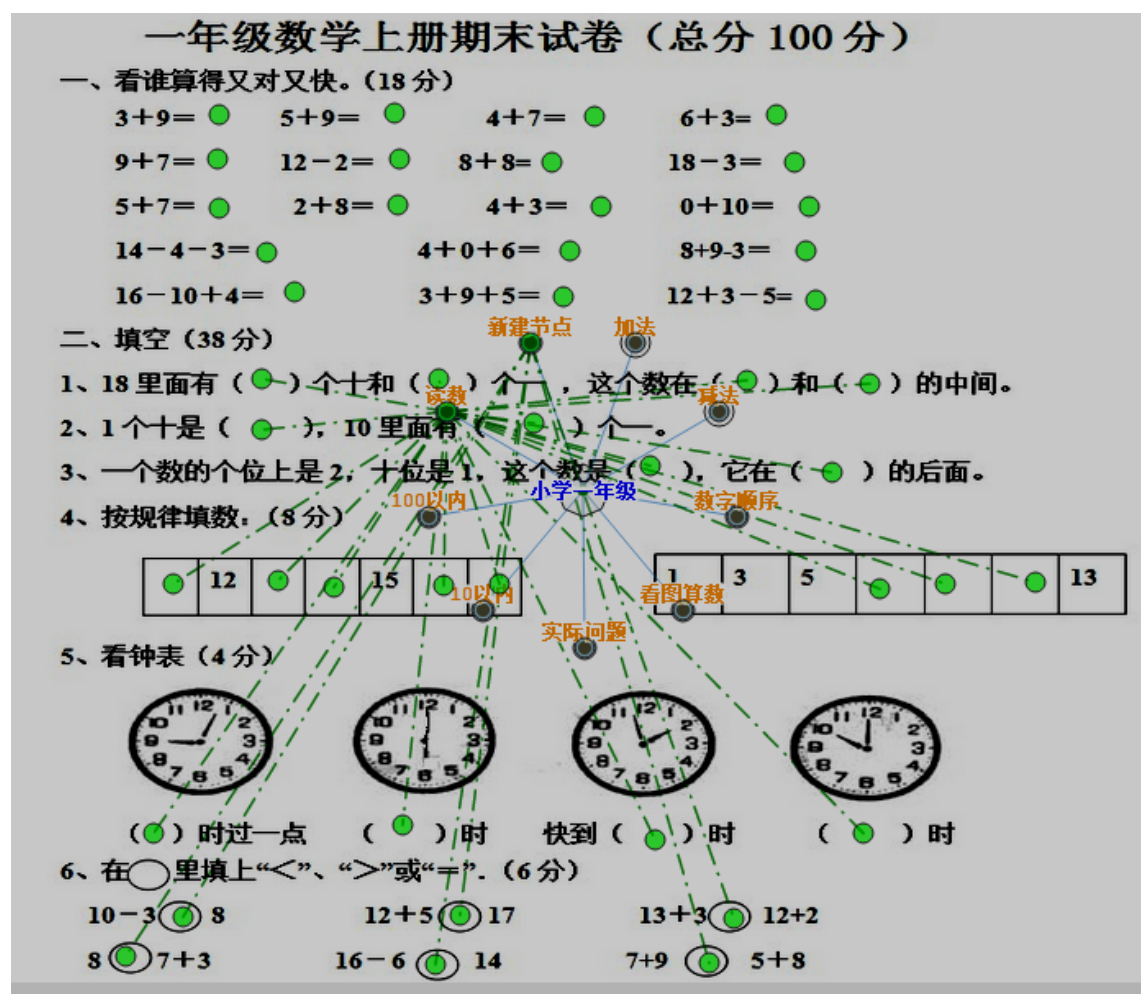

Fig. (12). Construction of a tree topology based on the topological space of test points.

(1) Vectorization of testing situation based on the COM frame: We scan the test paper in order to establish the human-machine testing situation, as shown in Fig. (12). We mark the places where answers are to be written in order to collect students' testing process and result. For example, mark on the related positon of " $5+9$ " and let students input relevant data using secondary windows, as shown in Fig. (11), which are then collected and recorded by the computer. Whether 13 or 31 is input, we will collect it as the test data of this problem. In the collected data, a sequence of cognitive symbols are collected based on the COM frame in order to form set $\mathrm{Q}$ and construct the topology $(\mathrm{Q}, \tau)$ of the test problem.
(2) The tree-based knowledge topology: According to the test objective and on the basis of the set of test points of the paper, we look for an equivalence class $\mathrm{Kn}$ in $\tau$ and define a quotient topology $\Phi$. Related test points form an equivalence class, are abstracted into a second-order knowledge structure set of test points, and further form a second-order topological space (K, $\Phi)$, as shown in Fig. (12).

In the above perspective, we demonstrate an analytical method based on the BSM digital environment and the tree map and realize the information observation of learning and examination processes and behaviors. We also build an abstract topological space of knowledge based on the 
observed data so as to visualize the problem space. All this provides empirical bases for teacher's teaching and offers references for teaching decisions.

\section{CONCLUSION}

Observing problem spaces based on coupled humanmachine cognition can obtain refine information about problem spaces and realize automated observation. It is the trend of the methods and technology for cognitive psychological studies. According to the manifold theory, a problem space is a high-dimensional cognitive manifold and it is crucial to look for low-dimensional space structure for dimensionality reduction. We consider that the lowdimensional structure of problem spaces is a tree structure.

After the construction of problem topology models, mathematical methods can be used to study problem spaces, establish the vector models of problem spaces, establish basic concepts such as problem objects, the size, angle, and vector addition and subtraction of objects, and even the process-oriented analysis of problem search and cognitive orbits.

Next, we will deepen the observation methods for problem spaces, further explore the refine structure of topological problem spaces, carry out dynamic studies on problem spaces, and predict the orbits of problem spaces.

\section{ABOUT THE AUTHORS}

First Author: Liu Changyong, master degree in Technology of Computer Application from Fuzhou University. 11 papers had been published.

Second Author: Cheng Rengui, master degree in Computer software and theory from Northwest University. 24 papers had been published.

Third Author: Meng Shimin, more than 10 papers on cognitive coupling states and cognitive digital manifold.

\section{CONFLICT OF INTEREST}

The authors confirm that this article content has no conflict of interest.

\section{ACKNOWLEDGEMENTS}

The paper is supported by the following funds: the Education Ministry Fund in Fujian Province (JA12321, JA13318) and The Special Fund for Industry Development and Scientific Research in Nanping, Fujian (N2011WZ04) and the Key Scientific Project Fund in Nanping, Fujian (N2012Z06-7).

\section{REFERENCES}

[1] A. Newell, and H. A. Simon, The theory of human problem solving, CA: Morgan Kaufmann Publishers, Inc, 1988, pp.33-51.

[2] G. J. Feist, E. Rosenberg, Psychology: Making Connections, NY: The McGranite Hill Publishers, 2009, pp.36-50.

[3] S. Yang, L. Guo, W. S. Yu, C. Y. Liu, and S. Meng, "Learning dynamics analysis based on computer-human interaction," Int. $J$. Emerg. Technol. Learning, vol. 8, pp. 5, 32-36, 2013.

[4] S. Yang, W. S. Yu, and C. Y. Liu, "A cognitive topology structure observation approach based on man-machine coupling states," Comput. Modell. New Technol., vol.17, no. 4, pp. 174-184, 2013.

[5] S. M. Meng, R. G. Cheng, and Y. L. Liu, "Cognitive topology mapping based on human-computer interaction," J. Internet Tech. Secured Transact., vol. 1, pp. 123-130, 2012.

[6] S. M. Meng, and C. Y. Liu, "The cognitive digital manifold based on the man-machine interactions," Comput. Inform. Tech., vol. 1, pp. 1-8, 2013. (in Chinese).

[7] S. Yang, C. Y. Liu, R. G. Cheng, W. Yu, S. Meng, Z. Xiao, and L. Guo, "Vectorization methods of hci situations based on cognitive frame," J. Chongqing Univ. Tech. (Nat. Sci.), vol. 10, pp. 102-106, 2013. (in Chinese).

[8] Y. Liu, and S. M. Meng, "Information sequence of cognitiveoriented 1: 1 digital classroom," J. Changchun Teachers College, vol. 12, pp. 121-124, 2012.

[9] M. Shiming, "Visualization complex cognitive networks," In: $3^{\text {rd }}$ Int. Conf. e-Education, e-Business, e-Management and e-Learning IPEDR, vol. 27, Singapore, 2012, pp.1-5. 